\title{
ENSAYOS MECÁNICOS SOBRE MORTEROS CON AGREGADOS DE PLÁSTICO RECICLADO DOSIFICADOS SEGÚN MODELO DE CONDUCTIVIDAD TÉRMICA
}

\author{
Mechanical tests on mortars with recycled plastic aggregates dosed under a model of thermal conductivity
}

\author{
Juan Pablo OJEDA*, Irma Teresa MERCANTE y Nicolás Horacio FAJARDO
}

Centro de Estudios de Ingeniería de Residuos Sólidos, Instituto de Medio Ambiente, Facultad de Ingeniería, Universidad Nacional de Cuyo, Centro Universitario (M5502KFA), Casilla de correo 405, Mendoza, Argentina

*Autor para correspondencia: juanpabloojedadangelo@hotmail.com

(Recibido: noviembre 2018; aceptado: agosto 2019)

Palabras clave: aislamiento térmico, residuos sólidos, materiales de construcción, mezclas cementicias

\section{RESUMEN}

Los residuos de plástico constituyen el $10 \%$ de los residuos sólidos a nivel mundial. El uso de agregados de plástico en el hormigón mejora algunas propiedades como el aislamiento térmico y el peso específico, aunque produce disminución de la resistencia a la compresión. En este trabajo se dosificaron mezclas con agregados de plástico reciclado de origen residual para satisfacer requisitos de aislamiento térmico. Para ello se desarrolló un modelo simplificado, calculando los espesores de plástico requeridos en un muro equivalente de $18 \mathrm{~cm}$ de espesor. Luego se moldearon probetas y se ensayaron a flexión y compresión. Los resultados confirmaron que la incorporación de plástico produjo una caída de la resistencia a la compresión y la flexión, excepto en el caso de gránulos (pellets). Se requirió un espesor teórico de plástico — con el modelo de muro multicapa propuesto - de 2.4 a $6 \mathrm{~cm}$ para lograr el aislamiento térmico deseado, frente a los $37 \mathrm{~cm}$ que hubiese requerido un muro realizado exclusivamente con mortero. Sin embargo, sólo una mezcla alcanzó la resistencia mínima requerida para la construcción de paneles. Se concluyó que los agregados redondeados exhibieron mejor comportamiento que los agregados con forma de hojuelas y que el modelo utilizado para la dosificación de agregados de plástico debería ser mejorado y contrastado con resultados experimentales en un trabajo futuro.

Key words: thermal insulation, solid waste, construction materials, cementitious mixtures

\begin{abstract}
Plastic waste constitutes $10 \%$ of the solid waste worldwide. The use of plastic aggregates in concrete improves some properties such as thermal insulation and specific weight, although it produces a decrease in strength. In this work, mixtures with plastic aggregates of residual origin were dosed to satisfy thermal insulation requirements. For this purpose, a simplified model was made, calculating the required plastic thicknesses in an equivalent wall of $18 \mathrm{~cm}$ thick. Then, specimens were molded and tested by bending and compression. The results confirmed that the incorporation of plastic produced a drop in compressive and flexural strength, except in the case of pellets. Under the proposed multilayer wall model, a theoretical thickness of plastic between 2.4 and $6 \mathrm{~cm}$ was required to achieve the desired thermal insulation, compared to $37 \mathrm{~cm}$
\end{abstract}


that a wall exclusively made of mortar would have required. However, only one mix reached the minimum strength required for the construction of panels. It was concluded that the rounded aggregates exhibited better behavior than the leaf-shaped aggregates and that the model used for the dosage of plastic aggregates should be improved and contrasted with experimental results in a future paper.

\section{INTRODUCCIÓN}

Según la Asociación Internacional de Residuos Sólidos (ISWA, por sus siglas en inglés), el volumen anual de residuos plásticos comercializados a nivel mundial representó menos del $5 \%$ de la producción de plásticos nuevos en 2012 (ISWA 2014). Sin embargo, esta situación está empezando a cambiar, como se aprecia en una de las estrategias claves del paquete de nuevas medidas sobre economía circular de la Unión Europea lanzada en diciembre de 2015. En dicha estrategia se destaca la importancia de abordar problemas de reciclabilidad, biodegradabilidad y presencia de sustancias peligrosas en los plásticos (CUE 2015).

Una alternativa para el reciclaje del plástico es la elaboración de mezclas cementicias, las cuales producen múltiples beneficios, como la valorización y el aprovechamiento de residuos sólidos, la sustitución de agregados naturales y las mejoras en algunas propiedades del material (menor peso específico y mejores condiciones aislantes) (Mercante et al. 2017).

Diferentes autores han estudiado la incorporación de agregados de distintos tipos de plásticos en morteros y hormigones. Frigione (2010) estudió el uso de polietileno tereftalato (PET) molido en reemplazo de agregado fino en un $5 \%$. El material se obtuvo de botellas residuales sin lavar. Se elaboraron probetas con diferentes contenidos de cemento y relación agua/cemento. Los ensayos se ejecutaron a los 28 y 365 días. Se encontró que los hormigones con incorporación de PET tuvieron similar trabajabilidad que las muestras de control; resistencia a la compresión y a la tracción un poco menores y mayor ductilidad.

Ismail et al. (2008) utilizaron plástico de origen residual como reemplazo parcial de arena. Construyeron probetas cúbicas para ensayos de resistencia a la compresión y densidad en seco, y probetas prismáticas para ensayos de resistencia a la flexión e índices de tenacidad. Los resultados demostraron que los valores de resistencia a la compresión y flexión de todas las mezclas tendieron a disminuir con el incremento del contenido de agregado de plástico reciclado en todas las edades. Los autores lo atribuyeron a la baja resistencia adhesiva entre la superficie del residuo de plástico y la pasta de cemento.
Casanova-del Ángel y Vázquez-Ruiz (2012) utilizaron PET obtenido de botellas de bebidas en reemplazo del agregado grueso pétreo. Con una granulometría adecuada se obtuvieron hormigones de menor peso específico, pero resistencia similar a los tradicionales. La relación agua/cemento fue menor para estos hormigones. Encontraron que es mejor emplear contenidos de cemento menores a $300 \mathrm{~kg} / \mathrm{m}^{3}$.

Batayneh et al. (2007) utilizaron plásticos y vidrio provenientes de residuos de construcción y demolición para reemplazar agregados en el hormigón. Si bien no especificaron qué tipo de plástico utilizaron, concluyeron que el agregado plástico provocó la caída de la resistencia a la compresión y de la resistencia a la flexión.

Choi et al. (2005) analizaron las propiedades mecánicas de mezclas con utilización de granulado de residuos de botellas plásticas de PET y escoria granulada de alto horno. Los calentaron juntos, quedando la escoria en la superficie de las partículas. La resistencia a la compresión y la densidad del hormigón disminuyeron a medida que creció la tasa de sustitución, pero la trabajabilidad del hormigón se vio mejorada.

En Argentina se han realizado estudios acerca del reemplazo de árido natural por partículas de plástico para la elaboración de mezclas cementicias destinadas a la fabricación de tejas y ladrillos (Gaggino y Argüello 2014) con buenos resultados. Además, en el mercado local es posible comprar agregados de poliestireno (PS) expandido, usado principalmente para la construcción de contrapisos no estructurales y mezclas preparadas para revoques aislantes.

Por otra parte, existen investigaciones sobre el uso de distintos materiales para conseguir aislamiento térmico en viviendas. Por ejemplo, Charca et al. (2015) estudiaron el uso de Stipa ichu, un tipo de pasto andino, como aislación térmica, concluyendo que se consigue un excelente aislamiento térmico a un bajo costo.

Chikhi et al. (2013) utilizaron fibras de Phoenix dactylifera en mezclas de yeso, concluyendo que el incremento del contenido de fibras produjo mayor aislamiento térmico. 
San-Juan et al. (2018) analizaron el uso de PS expandido para fabricar probetas y medir la conductividad de mezclas elaboradas con PS reciclado y con PS comercial, virgen. Encontraron que cuando se consigue mejor compacidad en la mezcla (usando partículas grandes y pequeñas de PS) se obtiene la mayor aislación térmica.

Viegas et al. (2016) hicieron una valoración cualitativa y cuantitativa de la aislación térmica provista por varios materiales usados en viviendas precarias. Mediante un ensayo de placa caliente analizaron la conductividad térmica de cartón, trozos de planchas de PS expandido, paja de trigo, botellas plásticas vacías y tapadas, y bollos de polietileno (PE), obteniendo resultados satisfactorios para viviendas factibles de ser construidas con herramientas sencillas por los propios moradores.

La presente investigación tiene como objetivo evaluar el desempeño mecánico de morteros con agregados de plástico obtenidos por reciclaje de residuos sólidos y dosificados según requisitos de aislación térmica.

El trabajo se enmarca en una investigación solicitada por una empresa local (Mendoza, Argentina) que construye viviendas prefabricadas para barrios sociales. Las mezclas serán utilizadas para la producción de paneles, por lo que deben cumplir requisitos de confort térmico de la norma 11605 del Instituto Argentino de Normalización y Certificación (IRAM, 1996) y de resistencia del reglamento del Centro de Investigación de los Reglamentos Nacionales de Seguridad para las Obras Civiles (CIRSOC) 202 (INTI 1982). También se destaca el aspecto social del plástico reciclado que se utiliza en esta investigación, el cual provino de organizaciones locales dedicadas al reciclaje y que dan empleo formal a personas en situación de vulnerabilidad socioeconómica.

\section{MATERIALES Y MÉTODOS}

En esta sección se describirán en primer lugar los materiales usados para la elaboración de los especímenes experimentales (probetas) y sus características. A continuación, en la parte metodológica, se hará la distinción entre la parte teórica y de cálculo y la parte experimental. Dentro de esta última se tratarán los ensayos de determinación de la densidad de los agregados de plástico y los ensayos mecánicos de flexión y compresión sobre probetas prismáticas y cúbicas.

Los materiales utilizados en la elaboración de las mezclas fueron:
- Dos tipos de arenas naturales silíceas de origen aluvial (denominadas arena fina y arena gruesa) en una proporción fija de $30 \%$ de arena fina y 70 $\%$ de arena gruesa. En el cuadro I se presentan el tamaño máximo nominal (TMN) y el módulo de fineza (MF) de las arenas.

CUADRO I. CARACTERÍSTICAS DE LAS ARENAS

\begin{tabular}{lll}
\hline & TMN $(\mathrm{mm})$ & MF \\
\hline Arena fina & 0.59 & 1.5 \\
Arena gruesa & 2.4 & 2.7 \\
\hline
\end{tabular}

TMN: tamaño máximo nominal del agregado, MF: módulo de finura

- Cemento Portland compuesto CPC 40, obtenido por la molienda conjunta de clínker Portland, puzolana natural, relleno (filler) calcáreo, yeso y aditivos.

- Agregados de plástico de PET, polipropileno (PP) y polietileno de baja densidad (PEBD). En el cuadro II pueden observarse los tipos de plástico utilizados, el origen de estos residuos, los procesos de transformación que sufrieron y las características del material resultante. En la figura 1 pueden observarse las muestras junto a una escala graduada cada $1 \mathrm{~cm}$.

- Agua potable.

\section{Parte metodológica teórica y de cálculo}

La dosificación se realizó siguiendo un modelo multicapa para muros de $18 \mathrm{~cm}$ de espesor que se describe más adelante en esta sección. La conductividad máxima adoptada para el muro fue de 1.85 $\mathrm{W} / \mathrm{m}^{2} \mathrm{~K}$, correspondiente a los requisitos de habitabilidad y confort higrotérmico de las viviendas en época invernal para la Provincia de Mendoza, Argentina.

El cálculo del volumen de plástico a dosificar se hizo considerándolo como si estuviera distribuido en una capa uniforme de $\mathrm{x} \mathrm{cm}$ de espesor $\mathrm{y}$ con base en las conductividades térmicas disponibles en el catálogo online de Goodfellow (2018) (Fig. 2). Las resistencias superficiales del mortero y la resistencia superficial interior y exterior se obtuvieron de la norma 11601 del IRAM (2002). Se destaca que éste es un modelo simplificado que no permite asegurar por sí solo que se conseguirá el aislamiento térmico buscado. El valor teórico de la cantidad de plástico calculado puede ser menor que el necesario debido a efectos de puente térmico. 
CUADRO II. AGREGADOS DE PLÁSTICO RECICLADO

\begin{tabular}{|c|c|c|c|c|c|c|c|c|c|c|c|}
\hline \multirow[b]{2}{*}{$\frac{\stackrel{\pi}{\Xi}}{\frac{\pi}{E}}$} & \multirow[b]{2}{*}{ 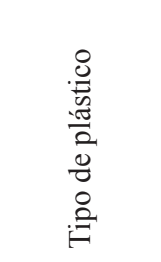 } & \multirow[b]{2}{*}{ 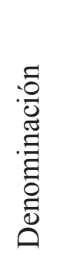 } & \multirow[b]{2}{*}{ 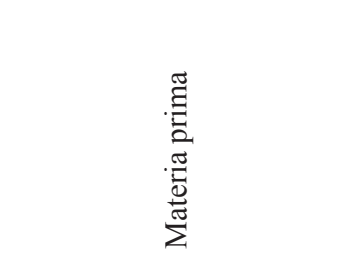 } & \multicolumn{6}{|c|}{ Proceso } & \multirow[b]{2}{*}{ 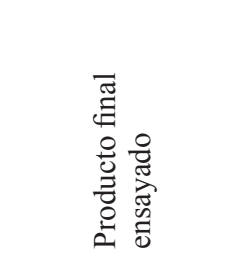 } & \multirow{2}{*}{ 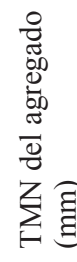 } \\
\hline & & & & 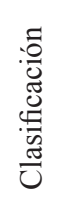 & نัّ & 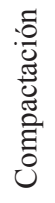 & 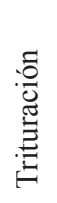 & 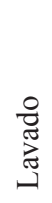 & 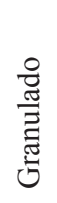 & & \\
\hline A & PP & $\mathrm{a}$ & $\begin{array}{l}\text { Restos de transformadores } \\
\text { y productos postconsumo } \\
\text { industriales }\end{array}$ & $x$ & $x$ & & $x$ & $x$ & $x$ & $\begin{array}{l}\text { Gránulos } \\
\text { redondeados. } \\
\text { Limpios }\end{array}$ & 2.4 \\
\hline \multirow[t]{2}{*}{ B } & PET & $\mathrm{b}$ & Botellas de bebidas & $x$ & & & $x$ & $x$ & & $\begin{array}{l}\text { Partículas lajosas. } \\
\text { Limpias }\end{array}$ & 4.8 \\
\hline & PP & $\mathrm{c}$ & $\begin{array}{c}\text { Tapitas y etiquetas } \\
\text { de botellas }\end{array}$ & $\times$ & & & $x$ & $x$ & & $\begin{array}{c}\text { Partículas lajosas. } \\
\text { Limpias }\end{array}$ & 4.8 \\
\hline $\mathrm{C}$ & PET y PP & $d$ & $\begin{array}{l}\text { Botellas y otros } \\
\text { recipientes }\end{array}$ & $\times$ & & $x$ & $\times$ & & & $\begin{array}{c}\text { Partículas lajosas. } \\
\text { Sucias }\end{array}$ & 9.5 \\
\hline $\mathrm{D}$ & PEBD y PP & $\mathrm{e}$ & Bolsas & $x$ & & & $x$ & & & $\begin{array}{c}\text { Partículas lajosas. } \\
\text { Sucias }\end{array}$ & 4.8 \\
\hline
\end{tabular}

TMN: tamaño máximo nominal del agregado, PP: polipropileno, PET: polietileno de tereftalato, PEBD: polietileno de baja densidad
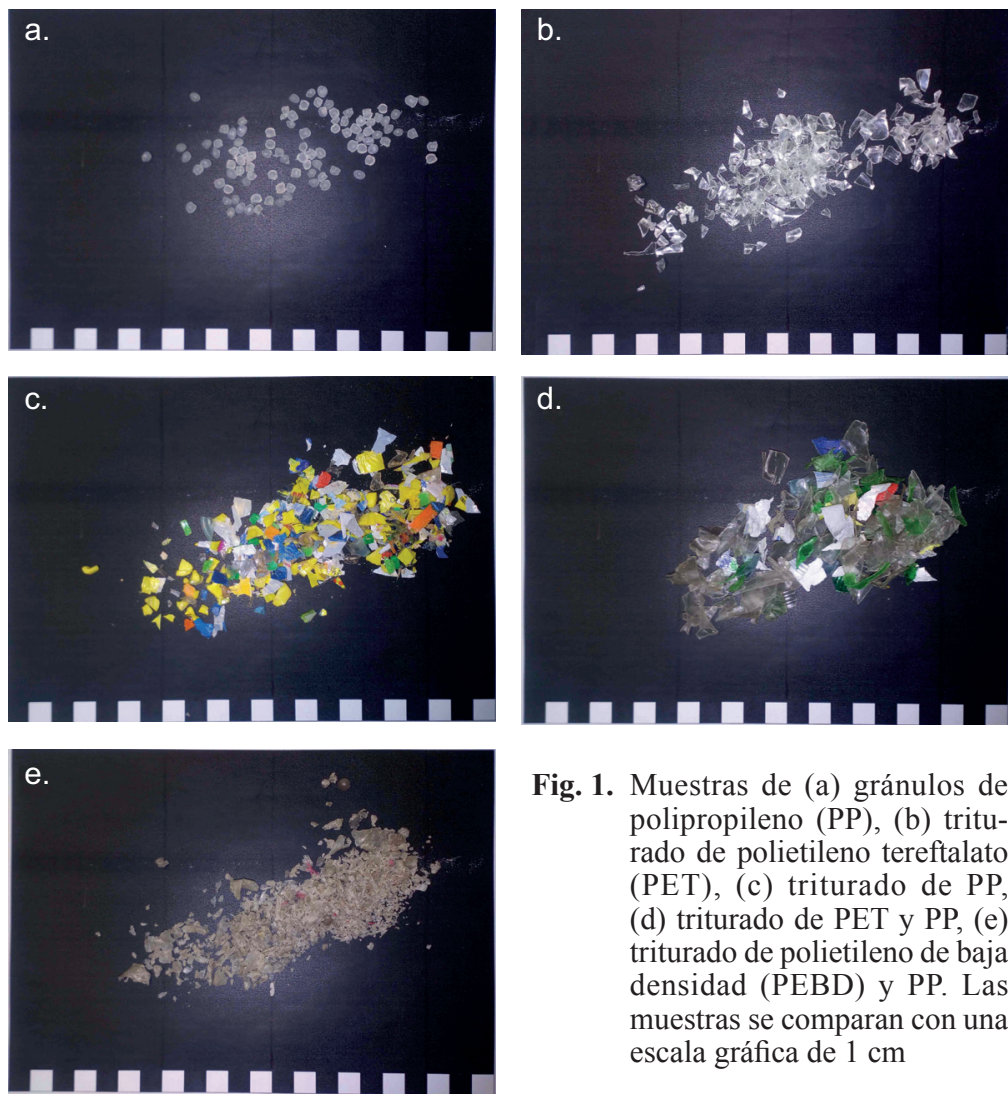

Fig. 1. Muestras de (a) gránulos de polipropileno (PP), (b) triturado de polietileno tereftalato (PET), (c) triturado de PP, (d) triturado de PET y PP, (e) triturado de polietileno de baja densidad (PEBD) y PP. Las muestras se comparan con una escala gráfica de $1 \mathrm{~cm}$ 


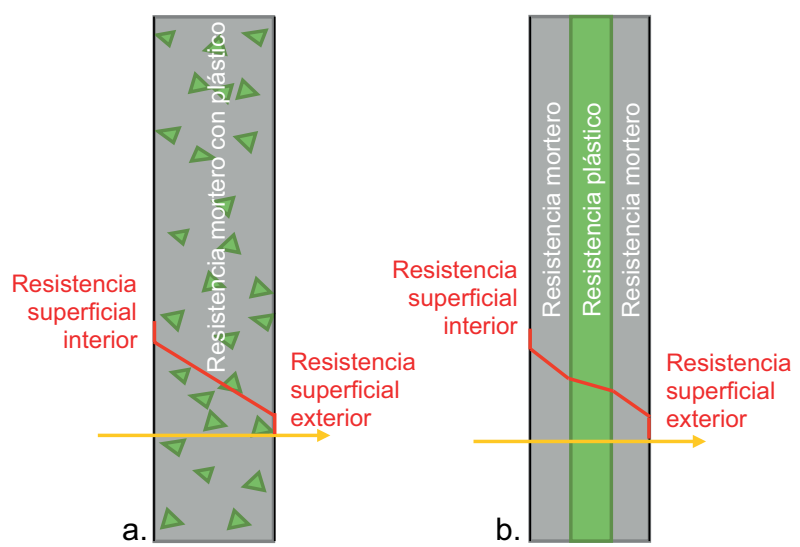

Fig. 2. transmisión de calor en (a) muro real con agregados de plástico y (b) modelo simplificado de cálculo con capa uniforme de plástico

$$
K \text { máx } a d m>\frac{1}{r s i+\frac{e-x}{\lambda m o r t e r o}+\frac{x}{\text { iplástico }}+r s e}
$$

donde $K$ máx $a d m$ es la conductividad máxima admisible en invierno $=1.85 \mathrm{~W} / \mathrm{m}^{2} \mathrm{~K}$; $r s i$ es la resistencia superficial interior $=0.13 \mathrm{~m}^{2} \mathrm{~K} / \mathrm{W}$; rse es la resistencia superficial exterior $=0.04 \mathrm{~m}^{2} \mathrm{~K} / \mathrm{W} ; e$ es el espesor del muro $=18 \mathrm{~cm} ; x$ es el espesor de plástico en el muro; $\lambda$ mortero es la conductividad del mortero (se adopta $1 \mathrm{~W} / \mathrm{m} \mathrm{K}$, obtenida de IRAM 2002), y $\lambda$ plástico es la conductividad del plástico (obtenida del catálogo Goodfellow 2018).

Se fijó una relación agua/cemento de 0.55 para todos los casos. Este valor fue un requisito de la empresa para obtener mezclas con suficiente trabajabilidad.

\section{Parte metodológica experimental}

Para caracterizar los agregados en cuanto a su densidad se utilizó una probeta graduada de laboratorio y se seleccionaron muestras de los agregados de plástico reciclado y agregados naturales para determinar las densidades. Se pesó el recipiente vacío $\left(M_{\mathrm{R}}\right)$ y luego el recipiente con la muestra de agregados $\left(M_{\mathrm{R}+\mathrm{A}}\right)$. Posteriormente se añadió un fluido de densidad conocida (agua destilada o etanol $96 \mathrm{vol}$ ) y se eliminaron los vacíos. Finalmente, se pesó el recipiente con agregados y agua $\left(M_{\mathrm{R}+\mathrm{A}+\mathrm{W}}\right)$ y se registró la medida del volumen ocupado por la mezcla $\left(V_{\mathrm{A}+\mathrm{w}}\right)$.

La densidad real se calculó como:

$\rho=\frac{M_{\mathrm{R}+\mathrm{A}}-M_{\mathrm{R}}}{V_{\mathrm{A}+\mathrm{W}}-\left(M_{\mathrm{R}+\mathrm{A}+\mathrm{W}}-M_{\mathrm{R}+\mathrm{A}}\right) / \rho_{f}}$

donde $\rho$ es la densidad real $\left[\mathrm{kg} / \mathrm{m}^{3}\right] ; M_{\mathrm{R}+\mathrm{A}}$ es la masa del recipiente con agregado $(\mathrm{kg}) ; M_{\mathrm{R}}$ es la masa del recipiente vacío $(\mathrm{kg}) ; M_{\mathrm{R}+\mathrm{A}+\mathrm{W}}$ es la masa del recipiente con agregado y agua $(\mathrm{kg}) ; V_{\mathrm{A}+\mathrm{W}}$ es el volumen de la mezcla de agua y agregado $\left(\mathrm{m}^{3}\right)$, y $\rho_{f}$ es la densidad del fluido empleado (agua $998 \mathrm{~kg} / \mathrm{m}^{3}$, etanol $\left.797 \mathrm{~kg} / \mathrm{m}^{3}\right)\left(\mathrm{kg} / \mathrm{m}^{3}\right)$.

También se realizaron ensayos mecánicos. Se confeccionaron probetas para ensayo de flexión y compresión a siete y 28 días. Los moldes empleados correspondieron a los establecidos en la norma 1622 del IRAM (2015). El curado se hizo sumergido en agua saturada con cal para asegurar una correcta hidratación del cemento y la consecuente ganancia de resistencia mecánica de las probetas. Los ensayos mecánicos se realizaron en una máquina universal de ensayos con capacidad máxima de 30000 kgf y resolución de $10 \mathrm{kgf}$.

Para cada edad de ensayo se elaboraron tres probetas prismáticas de $4 \times 4 \times 16 \mathrm{~cm}$ y se ensayaron a flexión (Fig. 3). Durante el ensayo se sometió la probeta a una carga gradual y localizada en la mitad de su luz hasta su rotura.

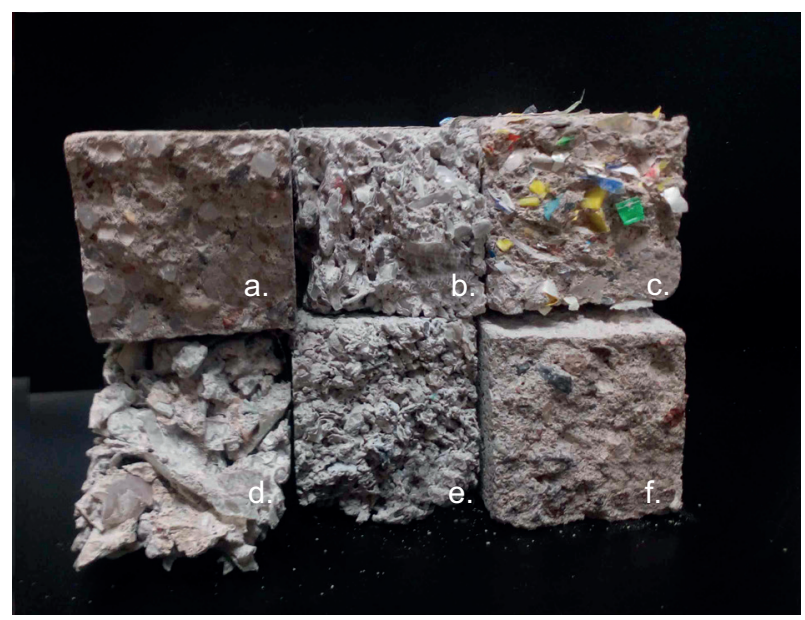

Fig. 3. Probetas prismáticas postensayo de mezclas con (a) gránulos de polipropileno (PP), (b) triturado de polietileno de tereftalato (PET), (c) triturado de PP, (d) triturado de PET y PP, (e) triturado de polietileno de baja densidad (PEBD) y PP, (f) mezcla patrón

Se determinó la resistencia a la flexión mediante la siguiente fórmula, obtenida de la norma $1622 \mathrm{del}$ IRAM (2015).

$R_{f}=\frac{1.5 \times F_{t} \times l}{b^{3}}$

donde $R_{f}$ es la resistencia a la flexión en MPa (megapascales); $F_{t}$ es la carga de rotura en $\mathrm{N}$; $l$ es la luz de ensayo en mm, y $b$ es el ancho de la probeta en $\mathrm{mm}$. 
Las seis mitades de probetas ensayadas a flexión también fueron ensayadas a compresión para cada edad. Asimismo, se ensayaron a compresión tres probetas cúbicas de $7 \times 7 \times 7 \mathrm{~cm}$ a los 28 días (Fig. 4). Con las mitades de las probetas a flexión a siete y a 28 días se buscó observar el crecimiento de la resistencia con el tiempo.

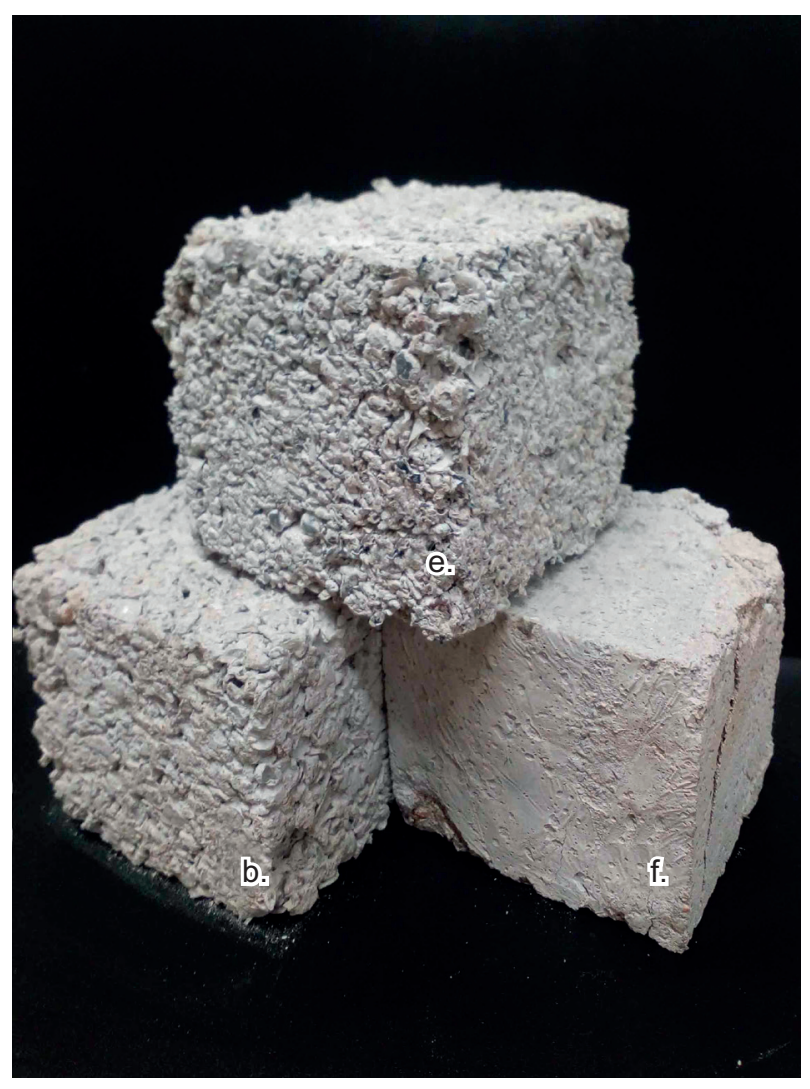

Fig. 4. Probetas cúbicas postensayo de mezclas con (e) triturado de polietileno de baja densidad (PEBD) y polipropileno (PP), (b) triturado de polietileno de tereftalato (PET), (f) mezcla patrón

Durante el ensayo, mediante la máquina universal, se aplicaron cargas de manera gradual y uniforme según lo establecido en la norma 1622 del IRAM (2015). En el caso de la probeta prismática, se empleó un dispositivo para aplicar la carga exclusivamente en un área de $4 \times 4 \mathrm{~cm}$ y en la cúbica se utilizó toda el área.

Se determinó la resistencia a la compresión a través de la siguiente fórmula.

$R_{c}=\frac{F_{c}}{A}$ donde $R_{c}$ es la resistencia a la compresión en MPa (megapascales); $F_{c}$ es la carga de rotura en N, y $A$ es el área determinada de cada probeta en $\mathrm{mm}^{2}$.

\section{RESULTADOS}

Los resultados de la determinación de la densidad de los plásticos se presentan en el cuadro III. Los mismos se utilizaron para calcular la dosificación de las distintas mezclas obteniendo un espesor de plástico en el muro equivalente y su dosificación asociada. Si se compara con un muro de mortero sin plástico, este debería poseer un espesor de $37 \mathrm{~cm}$ para conseguir una conductividad de $1.85 \mathrm{~W} / \mathrm{m}^{2} \mathrm{~K}$.

CUADRO III. ESPESORES EQUIVALENTES PARA CADA AGREGADO, DENSIDAD Y DOSIFICACIÓN EQUIVALENTE

\begin{tabular}{|c|c|c|c|c|}
\hline 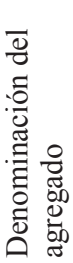 & 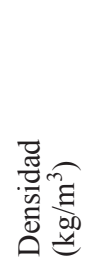 & 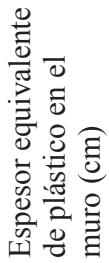 & 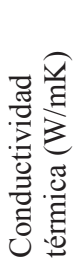 & 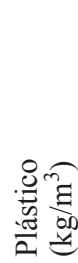 \\
\hline $\mathrm{a}$ & 934 & 2.4 & 0.11 & 118.5 \\
\hline b & 1092 & 4.5 & 0.19 & 266.8 \\
\hline $\mathrm{c}$ & 925 & 2.4 & 0.11 & 118.3 \\
\hline d & 1060 & 3.6 & 0.16 & 211.1 \\
\hline e & 930 & 6.0 & 0.24 & 308.2 \\
\hline
\end{tabular}

Cada tipo de plástico, según su conductividad térmica, requirió un espesor equivalente al del modelo mostrado en la figura $\mathbf{2 b}$.

Los agregados denominados (b) y (d) (de acuerdo con el cuadro II y la figura 1), presentaron similitud en sus pesos específicos, aunque (b) se compone exclusivamente de PET y (d) presenta mezcla de PET y PP. Otro grupo con similares densidades fue el de los plásticos denominados (a), (c) y (e); aunque (a) y (c) son exclusivamente PP y (e) es una mezcla de PEBD y PP.

En el cuadro IV figuran las dosificaciones en peso de las mezclas, con la cantidad de arena fina, arena gruesa, cemento, agua y plásticos. Se mantuvo fija la relación entre el agua y el cemento. Se aclara que la muestra patrón es aquella que no posee plástico.

Con las resistencias a flexión obtenidas de la fórmula mencionada anteriormente, se calculó un promedio de las resistencias a siete y 28 días. En el cuadro $\mathrm{V}$ se observan las resistencias mencionadas. 
CUADRO IV. DOSIFICACIONES DE LAS MEZCLAS

\begin{tabular}{cccccc}
\hline $\begin{array}{c}\text { Denominación } \\
\text { de la mezcla }\end{array}$ & $\begin{array}{c}\text { Arena Fina } \\
\left(\mathrm{kg} / \mathrm{m}^{3}\right)\end{array}$ & $\begin{array}{c}\text { Arena Gruesa } \\
\left(\mathrm{kg} / \mathrm{m}^{3}\right)\end{array}$ & $\begin{array}{c}\text { Cemento } \\
\left(\mathrm{kg} / \mathrm{m}^{3}\right)\end{array}$ & $\begin{array}{c}\text { Agua } \\
\left(\mathrm{kg} / \mathrm{m}^{3}\right)\end{array}$ & $\begin{array}{c}\text { Plástico } \\
\left(\mathrm{kg} / \mathrm{m}^{3}\right)\end{array}$ \\
\hline Patrón & 469.6 & 1082.9 & 367.8 & 202.3 & 0 \\
a & 375.7 & 866.4 & 376.9 & 207.3 & 118.5 \\
b & 304.8 & 702.8 & 359.2 & 197.6 & 266.8 \\
c & 375.3 & 865.4 & 376.5 & 207.1 & 118.3 \\
d & 241.1 & 556.0 & 284.2 & 156.3 & 211.1 \\
e & 41.5 & 95.8 & 245.1 & 134.8 & 308.2 \\
\hline
\end{tabular}

CUADRO V. RESISTENCIAS A LA FLEXIÓN (Rf)

\begin{tabular}{|c|c|c|c|c|c|}
\hline \multicolumn{2}{|c|}{ Denominación } & \multicolumn{2}{|c|}{$\mathrm{Rf}_{7}$ días $(\mathrm{MPa})$} & \multicolumn{2}{|c|}{$\mathrm{Rf}_{28 \text { días }}(\mathrm{MPa})$} \\
\hline \multirow{3}{*}{ Patrón } & 1 & 1.97 & 1.90 & - & \multirow{3}{*}{2.08} \\
\hline & 2 & 1.97 & & 2.19 & \\
\hline & 3 & 1.75 & & 1.97 & \\
\hline \multirow{3}{*}{$\mathrm{a}$} & 1 & 1.86 & 2.23 & 3.07 & \multirow{3}{*}{2.85} \\
\hline & 2 & 2.63 & & 2.85 & \\
\hline & 3 & 2.19 & & 2.63 & \\
\hline \multirow{3}{*}{$\mathrm{b}$} & 1 & 0.42 & 0.35 & 0.20 & \multirow{3}{*}{0.24} \\
\hline & 2 & 0.42 & & 0.31 & \\
\hline & 3 & 0.20 & & 0.20 & \\
\hline \multirow{3}{*}{$\mathrm{c}$} & 1 & 1.16 & 1.45 & 0.64 & \multirow{3}{*}{0.79} \\
\hline & 2 & 1.37 & & 0.86 & \\
\hline & 3 & 1.81 & & 0.86 & \\
\hline \multirow{3}{*}{ d } & 1 & - & - & 0.20 & \multirow{3}{*}{0.20} \\
\hline & 2 & - & & - & \\
\hline & 3 & - & & - & \\
\hline \multirow{3}{*}{ e } & 1 & 0.09 & 0.09 & - & \multirow{3}{*}{0.15} \\
\hline & 2 & - & & 0.20 & \\
\hline & 3 & - & & 0.09 & \\
\hline
\end{tabular}

Nota: las celdas con ausencia de valores corresponden a probetas que no pudieron ensayarse a flexión porque se partieron antes del ensayo. Aun así, las mitades obtenidas se ensayaron a compresión MPa: megapascal

Las probetas patrón, (a) y (e) exhibieron ganancia de la resistencia a la flexión con el tiempo, mientras que (b) y (c) perdieron resistencia. Esto puede deberse a algún tipo de interacción desfavorable entre la mezcla y el plástico. Deberían hacerse más ensayos para obtener conclusiones al respecto.

En el caso de la mezcla (d), no pudo ensayarse a siete días, ya que las probetas se rompieron durante el desmolde y curado. La mezcla se caracterizó por ser poco cohesiva, sumamente porosa y poco trabajable.

Se obtuvieron tres resistencias a compresión para cada mezcla, como los promedios de las seis mitades de las probetas ensayadas a flexión a siete y 28 días, y de las tres probetas cúbicas a 28 días. En los cuadros VI y VII se observan las resistencias.

CUADRO VI. RESISTENCIAS A LA COMPRESIÓN (Rpc). PROBETAS PRISMÁTICAS

\begin{tabular}{|c|c|c|c|c|c|}
\hline \multicolumn{2}{|c|}{ Denominación } & \multicolumn{2}{|c|}{$\mathrm{Rpc}_{7 \text { días }}(\mathrm{MPa})$} & \multicolumn{2}{|c|}{$\mathrm{Rpc}_{28 \text { días }}(\mathrm{MPa})$} \\
\hline \multirow{3}{*}{ Patrón } & 1 & 20.8 & \multirow{3}{*}{17.2} & 18.9 & \multirow{3}{*}{21.9} \\
\hline & 2 & 11.7 & & 25.2 & \\
\hline & 3 & 19.1 & & 21.5 & \\
\hline \multirow{3}{*}{$\mathrm{a}$} & 1 & 16.28 & \multirow{3}{*}{18.0} & 26.04 & \multirow{3}{*}{26.7} \\
\hline & 2 & 18.83 & & 27.43 & \\
\hline & 3 & 18.86 & & 26.50 & \\
\hline \multirow{3}{*}{ b } & 1 & 2.86 & \multirow{3}{*}{3.1} & 3.16 & \multirow{3}{*}{3.8} \\
\hline & 2 & 3.40 & & 4.09 & \\
\hline & 3 & 2.95 & & 4.21 & \\
\hline \multirow{3}{*}{$\mathrm{c}$} & 1 & 7.35 & \multirow{3}{*}{8.6} & 8.47 & \multirow{3}{*}{8.5} \\
\hline & 2 & 8.19 & & 7.97 & \\
\hline & 3 & 10.33 & & 9.02 & \\
\hline \multirow{3}{*}{ d } & 1 & 0.81 & \multirow{3}{*}{0.9} & 0.81 & \multirow{3}{*}{0.9} \\
\hline & 2 & 1.04 & & 1.04 & \\
\hline & 3 & 0.72 & & 0.72 & \\
\hline \multirow{3}{*}{$\mathrm{e}$} & 1 & 1.13 & \multirow{3}{*}{0.8} & 1.13 & \multirow{3}{*}{0.8} \\
\hline & 2 & 0.46 & & 0.46 & \\
\hline & & 0.69 & & 0.69 & \\
\hline
\end{tabular}

MPa: megapascal

En el caso de la resistencia a la compresión, las probetas mejoraron su desempeño con la edad (patrón, [a] y [b]), o bien, lo mantuvieron (casos [c], [d], [e]). Sin embargo, se esperaría una mayor ganancia de resistencia con la edad. En la mezcla patrón se logró el $79 \%$ de la resistencia de diseño a siete días, en la mezcla (a) el $67 \%$ y en la mezcla (b) el $82 \%$.

Los resultados obtenidos mostraron que la incorporación de plástico produjo disminución de la resistencia a compresión en casi todos los casos, 
CUADRO VII. RESISTENCIAS A LACOMPRESIÓN (Rcc). PROBETAS CÚBICAS

\begin{tabular}{|c|c|c|c|}
\hline \multicolumn{2}{|c|}{ Denominación } & \multicolumn{2}{|c|}{$\operatorname{Rcc}_{28}$ días $(\mathrm{MPa})$} \\
\hline \multirow{3}{*}{ Patrón } & 1 & 19.2 & \multirow{3}{*}{21.3} \\
\hline & 2 & 24.8 & \\
\hline & 3 & 20.0 & \\
\hline \multirow{3}{*}{$\mathrm{a}$} & 1 & 25.9 & \multirow{3}{*}{25.5} \\
\hline & 2 & 25.0 & \\
\hline & 3 & ---- & \\
\hline \multirow{3}{*}{$\mathrm{b}$} & 1 & 3.5 & \multirow{3}{*}{3.5} \\
\hline & 2 & 3.4 & \\
\hline & 3 & 3.6 & \\
\hline \multirow{3}{*}{$\mathrm{c}$} & 1 & 11.8 & \multirow{3}{*}{11.1} \\
\hline & 2 & 9.6 & \\
\hline & 3 & 11.8 & \\
\hline \multirow{3}{*}{ d } & 1 & 0.6 & \multirow{3}{*}{0.6} \\
\hline & 2 & 0.6 & \\
\hline & 3 & 0.6 & \\
\hline \multirow{3}{*}{ e } & 1 & 0.7 & \multirow{3}{*}{0.7} \\
\hline & 2 & 0.8 & \\
\hline & 3 & 0.7 & \\
\hline
\end{tabular}

Nota: la probeta a3 no pudo ser ensayada

Mpa: megapascal

excepto en el caso (a) que superó la resistencia patrón. En parte esto se debió a un mayor contenido de cemento y una buena trabajabilidad dada por la forma redondeada de los gránulos (pellets) de PP.

Las mayores resistencias a compresión fueron las de las mezclas (a) y (c). Estas tuvieron menor cantidad de plástico para cumplir con los requerimientos térmicos.

Los resultados del ensayo a compresión para probetas cúbicas dieron en general menores valores que aquellos para probetas prismáticas. Esto se debe al cambio de tamaño de la probeta (de 4 a $7 \mathrm{~cm}$ de alto), que en ambos casos fue de esbeltez 1 .

Para emplear estas mezclas en la elaboración de paneles se requiere una resistencia a la compresión de al menos $13 \mathrm{MPa}$. Se observa que la única dosificación propuesta que alcanza este requisito es la (a). En el caso de la mezcla (c), podría aumentarse el contenido de cemento para alcanzar la resistencia requerida. Las demás mezclas están lejos de satisfacer el requisito, pero podrían tener aplicaciones no estructurales.

Las mezclas con partículas provenientes de trituración tuvieron baja trabajabilidad y tendieron a ser porosas, mientras que la mezcla con gránulos fue más trabajable y compacta. Estos efectos se tradujeron en mayor resistencia para esta última mezcla (a). Sin embargo, desde el punto de vista ambiental, los gránulos son el tipo de plástico con más proceso de reciclaje; por lo tanto, con mayores cargas ambientales. En futuros ensayos mecánicos debería realizarse una mayor trituración de los agregados (b), (c), (d) y (e) para mejorar el desempeño de las mezclas.

\section{DISCUSIÓN}

Los ensayos planteados se hicieron sobre probetas prismáticas y cúbicas para mortero; sin embargo, en futuros ensayos se considera conveniente utilizar probetas cilíndricas de esbeltez 2. Esto permitiría obtener resultados más representativos y comparables con los disponibles en la bibliografía consultada.

Los resultados obtenidos a partir del modelo teórico arrojan una cantidad de plástico a dosificar que podría ser muy inferior a la necesaria para lograr la conductividad térmica requerida. Esto podría determinarse mediante ensayos de conductividad térmica para conocer los resultados empíricos de la investigación y validar o corregir las dosificaciones hechas a priori. De todos modos, los ensayos mecánicos indican que con estas cantidades de plástico no es posible alcanzar la resistencia deseada y con valores superiores se obtendrían caídas de resistencia aún mayores.

El modelo de muro multicapa propuesto no asegura el aislamiento térmico esperado, ya que no es asimilable a un material heterogéneo como el mortero con agregados de plástico. En el material real se producen puentes térmicos que rodean las partículas de plástico y, de esta manera, se pierde aislamiento.

Un modelo más exacto podría ser el siguiente:

$K$ máx $a d m>\frac{1}{r s i+\frac{e}{\text { imortero con plastico }}+r s e}$

donde $K$ máx $a d m$ es la conductividad máxima admisible en invierno $=1.85 \mathrm{~W} / \mathrm{m}^{2} \mathrm{~K} ; r s i$ es la resistencia superficial interior $=0.13 \mathrm{~m}^{2} \mathrm{~K} / \mathrm{W}$; rse es la resistencia superficial exterior $=0.04 \mathrm{~m}^{2} \mathrm{~K} / \mathrm{W}$, y $e$ es el espesor del muro $=18 \mathrm{~cm}$.

De lo anterior se obtiene que sería necesario lograr una conductividad térmica máxima en el material resultante ( $\lambda$ mortero con plástico) de: $0.49 \mathrm{~W} / \mathrm{m} \mathrm{K}$ :

El valor de $\lambda$ mortero con plástico puede obtenerse como:

$\begin{aligned} & \lambda \text { mortero } \\ & \text { con plástico }\end{aligned}=\frac{(1-x) * \lambda \text { mortero }+x * \lambda \text { plástico }}{1}$ 
donde $\lambda$ mortero es la conductividad del mortero solo $=1 \mathrm{~W} / \mathrm{m} \mathrm{K} ; \lambda$ plástico es la conductividad del plástico, y $x$ es el volumen de plástico en la mezcla total.

Este modelo arrojó los siguientes requerimientos de plástico para cada mezlca: en la mezcla (a) serían necesarios $535 \mathrm{~kg}$ de plástico por cada $\mathrm{m}^{3}$ de mezcla, en la (b) $688 \mathrm{~kg} / \mathrm{m}^{3}$, en la (c) $530 \mathrm{~kg} / \mathrm{m}^{3}$, en la (d) $644 \mathrm{~kg} / \mathrm{m}^{3}$ y en la (e) $624 \mathrm{~kg} / \mathrm{m}^{3}$. Estos valores son, en promedio, tres veces mayores a los calculados con el modelo simplificado.

\section{CONCLUSIONES}

Se realizaron dosificaciones con base en un modelo simplificado de muro multicapa para calcular la conductividad térmica teórica de muros de $18 \mathrm{~cm}$ de espesor. Se obtuvo un espesor de plástico necesario de 2.4 a $6 \mathrm{~cm}$, según el agregado analizado, mientras que un muro de mortero solo debería tener un espesor total de $37 \mathrm{~cm}$. Este modelo simplificado arrojó dosificaciones de plástico que pueden estar por debajo de las cantidades requeridas para lograr la aislación deseada, debido a que en el material heterogéneo real se forman puentes térmicos en el mortero que rodea a las partículas de plástico. Un modelo más realista triplicó las cantidades de plástico requeridas por el modelo simplificado multicapa.

El agregado que mostró mejor desempeño mecánico fue el de gránulos de PP. Esto se debió a su forma redondeada, que es más beneficiosa que la forma de hojuela que tuvo el resto de los agregados. A su vez, ésta fue la única mezcla que superó la resistencia mínima requerida de $13 \mathrm{MPa}$ para poder aplicarse en la construcción de paneles. Considerando esto, dosificaciones con mayor contenido de agregado plástico reducirían aún más la resistencia mecánica del material resultante, por lo que no podría utilizarse para los fines estructurales que se buscan. Las mezclas obtenidas podrían tener otras aplicaciones no estructurales.

El resto de los agregados de plástico reciclado podría someterse a mayor tiempo de trituración para obtener partículas más regulares en todas sus dimensiones, evitando la forma de hojuelas que genera planos de discontinuidad en el material. Esto a su vez produciría una mejora en la trabajabilidad de la mezcla fresca.

En una próxima etapa sería conveniente realizar ensayos mecánicos sobre probetas cilíndricas y ensayos de conductividad térmica para validar o corregir las dosificaciones hechas a priori por requisitos de conductividad térmica con el modelo simplificado.

\section{REFERENCIAS}

Batayneh M., Marie I. y Asi I. (2007). Use of selected waste materials in concrete mixes. Waste Manage. 27 (12), 1870-1876.

DOI: 10.1016/j.wasman.2006.07.026

Casanova-del Ángel F. y Vázquez-Ruiz J.L. (2012). Manufacturing light concrete with PET aggregate. International Scholarly Research Network Civil Engineering 2012, 287323. DOI: 10.5402/2012/287323

Charca S., Noel J., Andia D., Flores J., Guzman A., Renteros C. y Tumialan J. (2015). Assessment of Ichu fibers as non-expensive thermal insulation system for the Andean regions. Energ. Buildings. 108, 55-60. DOI: 10.1016/j.enbuild.2015.08.053

Chikhi M., Agoudjil B., Boudenne A. y Gherabli A. (2013). Experimental investigation of new biocomposite with low cost for thermal insulation. Energ. Buildings. 66, 267-273. DOI: 10.1016/j.enbuild.2013.07.019

Choi Y.W., Moon D.J., Chung J.S. y Cho S.K. (2005). Effects of waste PET bottles aggregate on the properties of concrete. Cement Concrete Res. 35 (4), 776-781.

DOI: 10.1016/j.cemconres.2004.05.014

CUE (2015). Cerrar el círculo: la Comisión adopta un ambicioso paquete de nuevas medidas sobre la economía circular para impulsar la competitividad, crear empleo y generar crecimiento sostenible. Comisión Unión Europea [en línea]. http://europa.eu/rapid/pressrelease IP-15-6203 es.htm 12/11/2018.

Frigione $\bar{M}$. (2010). Recycling of PET bottles as fine aggregate in concrete. Waste Manage. 30 (6), 1101-1106. DOI: 10.1016/j.wasman.2010.01.030

Gaggino R. y Argüello R. (2014). Building components made from recycled plastics. Key Eng. Mat. 600, 615615. DOI: 10.4028/www.scientific.net/KEM.600.615

Goodfellow (2018). Catálogo de polímeros - propiedades térmicas. Catálogo. Goodfellow. Cambridge, Inglaterra, $28 \mathrm{pp}$.

ISWA (2014). Globalisation and Waste Management. International Solid Waste Association. Viena, Austria, $55 \mathrm{pp}$.

INTI (1982). Centro de Investigación de los Reglamentos Nacionales de Seguridad para las Obras Civiles (CIRSOC) 202. Hormigón Liviano de Estructura Compacta. Instituto Nacional de Tecnología Industrial. Ciudad Autónoma de Buenos Aires, Argentina, 89 pp.

IRAM (1996). Norma 11605. Acondicionamiento térmico de edificios. Condiciones de habitabilidad en edificios. Valores máximos de transmitancia térmica en cerramientos opacos. Instituto Argentino de Normalización y Certificación. Ciudad Autónoma de Buenos Aires, Argentina, 27 pp. 
IRAM (2002). Norma 11601. Aislamiento térmico de edificios. Método de cálculo. Propiedades térmicas de los componentes y elementos de construcción en régimen estacionario. Instituto Argentino de Normalización y Certificación. Ciudad Autónoma de Buenos Aires, Argentina, $52 \mathrm{pp}$.

IRAM (2015). Norma 1622. Cemento portland. Determinación de resistencias mecánicas. Instituto Argentino de Normalización y Certificación. Ciudad Autónoma de Buenos Aires, Argentina, 25 pp.

Ismail Z.Z. y AL-Hashmi E.A. (2008). Use of waste plastic in concrete mixture as aggregate replacement. Waste Manage. 28 (11), 2041-2047.

DOI: $10.1016 /$ j.wasman.2007.08.023

Mercante I., Ojeda J., Alejandrino C. y Chini J. (2017). Revisión bibliográfica: Hormigón con agregados de plástico reciclados. Memorias. VII Simposio Iberoamericano en Ingeniería de Residuos, Santander, España. 13 y 14 de junio, 136-141.
San-Juan G.A., Viegas G.M. y Jodra J.I. (2018). Aislación térmica alternativa, reutilizando poliestireno expandido de descarte, orientado a las necesidades de cooperativas de reciclado. Memorias. X Congreso Regional de Tecnología en Arquitectura (CRETA). La Plata, Argentina. 29, 30 y 31 de agosto, 391-400.

Viegas G. M., Walsh C. y Barros M.V. (2016). Evaluación cuali-cuantitativa de aislaciones térmicas alternativas para viviendas: el caso de la agricultura familiar. Revista INVI 31 (86), 89-117.

DOI: $10.4067 / \mathrm{S} 0718-83582016000100004$ 